\title{
Inyección epidural de betametasona en el síndrome radicular lumbosacro: evaluación de su eficacia por abordajes interlaminar y transforaminal
} Epidural betamethasone injection in lumbosacral radicular syndrome: efficacy evaluation for interlaminar and transforaminal approaches

\author{
P. Castromán¹, M. Surbano², S. Ayala², A. Schwartzmann², S. Castelli y G. Varaldi
}

${ }^{1}$ Profesor Agregado. Servicio de Terapia del Dolor. Departamento y Cátedra de Anestesiología. Hospital de Clínicas. Montevideo, Uruguay. ${ }^{2} P r o f e s o r$ Adjunto. Servicio de Terapia del Dolor. Departamento y Cátedra de Anestesiología. Hospital de Clínicas. Montevideo, Uruguay. ${ }^{3}$ Anestesiólogo. Servicio de Terapia del Dolor. Departamento y Cátedra de Anestesiología. Hospital de Clínicas. Montevideo, Uruguay. ${ }^{4}$ Asistente. Servicio de Terapia del Dolor. Departamento y Cátedra de Anestesiología. Hospital de Clínicas. Montevideo, Uruguay

\section{ABSTRACT}

Introduction: Betamethasone is a frequently used steroid for epidural injection, recently incorporated in the Uruguayan pharmaceutical market.

Aims: The aim of this study is to evaluate the efficacy of parasagittal interlaminar and transforaminal epidural betamethasone in unilateral lumbosacral radicular syndrome, utilizing the Brief Pain Inventory (BPI).

Material and method: Is a prospective study comparing parasagittal interlaminar and transforaminal epidural betamethasone. Patients with chronic unilateral lumbosacral radicular pain were included. The BPI was administered before and one month after epidural injections. Pain intensity was measured by the Visual Numeric Scale (VNS, question 6 of the BPI) and the Intensity Score. Interference of pain in daily activities was measured by the Interference Score. Satisfactory responses to injections were considered with a 2 points reduction in VNS. The statistical evaluation was performed by paired an unpaired T test to continuous data and Chi Square to evaluate proportions. A p value less than 0.05 was considered statistically significant.

Results: Fifty four patients were treated with epidural betamethasone. In 29 the parasagittal interlaminar route was utilized while 25 were treated by the trans-
Castromán P, Surbano M, Ayala S, Schwartzmann A, Castelli S, Varaldi G. Inyección epidural de betametasona en el sindrome radicular lumbosacro: evaluación de su eficacia por abordajes interlaminar y transforaminal. Rev Soc Esp Dolor 2019;26(5):263-269

\section{RESUMEN}

Introducción: La betametasona es un esteroide utilizado en las inyecciones epidurales, de reciente incorporación en Uruguay.

Objetivos: Evaluar la eficacia de la inyección epidural de betametasona administrada por los abordajes interlaminar parasagital y transforaminal en pacientes con dolor radicular lumbosacro unilateral crónico, utilizando el Inventario Abreviado de Dolor.

Material y método: Estudio prospectivo aleatorizado que compara la administración de betametasona por ambos accesos, interlaminar parasagital y transforaminal. El IAD se aplicó previamente y al mes de las inyecciones epidurales. La intensidad del dolor se midió por la Escala Visual Numérica (EVN) obtenida de la pregunta 6 del IAD y el Índice de Intensidad. También se midió la interferencia funcional producida por el dolor mediante el Índice de Interferencia. Se estableció como respuesta satisfactoria un descenso de 2 puntos en la EVN basal al mes de los procedimientos, definiendo a estos pacientes como respondedores. Se utilizó la prueba t de Student y de Chi cuadrado para el análisis estadístico, tomando un valor de $p<0,05$ como significancia estadística.

Resultados: Un total de 154 pacientes recibieron betametasona epidural. En 29 de ellos se realizó un

Recibido: 28-07-2018

Aceptado: 19-03-2019

Correspondencia: Pablo Castromán pablocastro227@gmail.com 
foraminal route. A $20 \%$ reduction in baseline VNS was observed with the interlaminar route and $36 \%$ reduction with transforaminal approach. Intensity and Interference Scores were also reduced. This reductions were statistically significant when comparing to baseline data (paired t test) but differences between groups were not significant (unpaired t test). However the number of positive responses as defined above was greater in the transforaminal group, $64 \%$ versus $38 \%$ in the interlaminar group, statistically significant difference using the Chi Square analysis $(p=0.01)$.

In patients with positive responses, interlaminar and transforaminal betamethasone produce clinical and statistically significant reductions in pain intensity and interference, without difference between groups.

Conclusion: Epidural betamethasone produced a reduction in pain intensity and interference utilizing the BPI, by the two routes utilized to access the epidural space. Although no statistically differences were observed in this reductions between groups, the frequency of positive responses were higher when the drug is administered by the transforaminal route. Epidural interlaminar parasagittal betamethasone injection is a reasonable alternative to the transforaminal route, without the neurologic complications described utilizing this technique.

Key words: Epidural steroid injections, lumbar radicular pain, betamethasone. abordaje interlaminar parasagital y en 25 transforaminal. La betametasona administrada por la vía interlaminar parasagital redujo la puntuación en la EVN un $20 \%$, y por la vía transforaminal en un $36 \%$. Estas diferencias fueron estadísticamente significativas cuando se compararon los datos basales y los obtenidos al mes de los procedimientos (prueba t de Student para muestras pareadas), sin encontrarse diferencias estadísticamente significativas entre grupos (prueba t de Student para muestras independientes). Los Índices de Intensidad e Interferencia también fueron reducidos por la inyección epidural de esteroides, sin diferencias significativas entre ambos accesos. La frecuencia de respuestas satisfactorias fue mayor en el grupo tratado por vía transforaminal, 16/25 (64\%) frente a 11/29 (38 \%) en el grupo tratado por vía interlaminar, diferencia estadísticamente significativa, con un valor de $p=0,01$ (prueba de Chi cuadrado). En aquellos pacientes definidos como respondedores, la betametasona administrada por los dos abordajes produjo una reducción clínica y estadísticamente significativa del dolor y de su repercusión evaluados por el IAD, sin encontrarse diferencias significativas entre los abordajes.

Conclusión: La inyección de betametasona epidural administrada por vía interlaminar parasagital y transforaminal redujo la intensidad del dolor y su interferencia funcional en pacientes con dolor radicular crónico. Los abordajes interlaminar parasagital y transforaminal fueron similares en eficacia, aunque con una frecuencia de respuestas satisfactorias mayor en los pacientes tratados por vía transforaminal. La inyección interlaminar parasagital de esteroides es una alternativa válida a la vía transforaminal, sin sus riesgos neurológicos.

Palabras clave: Inyecciones epidurales de esteroides, dolor radicular lumbar, betametasona.

\section{INTRODUCCIÓN}

La inyección epidural de esteroides es uno de los procedimientos que utilizamos con más frecuencia en el tratamiento del dolor radicular lumbar en nuestro servicio (1). La betametasona de depósito es un esteroide de amplia utilización para aplicación epidural en el mundo, de reciente incorporación al vademécum de nuestro país (2-4).

La inyección de esteroides en el espacio epidural se realiza con más frecuencia a través de los espacios interlaminar o transforaminal (5). No existen evidencias científicas de que una vía de administración sea más efectiva que la otra, aunque existe la percepción de que la vía transforaminal logra mejores resultados, al llevar una concentración mayor de esteroide a la zona de conflicto discorradicular $[6,7)$. A pesar de esto, es conocido que la vía implica un riesgo potencial mayor de lesiones neurológicas, provocadas por isquemia medular secundaria a lesión de la arteria radiculomedular. Esta lesión se ha asociado a vasoespasmo u oclusión vascular; esto último podría estar relacionado con el tamaño de las partículas del esteroide inyectado (8). En este sentido, la betametasona de depósito es el este- roide que presenta las partículas de menor tamaño, y de este modo podría tener un perfil de seguridad más adecuado que la triamcinolona, el agente más utilizado para estas inyecciones en nuestro medio (9).

El objetivo de nuestro estudio fue evaluar la eficacia de la inyección epidural de betametasona bajo radioscopia, en pacientes con dolor radicular lumbar unilateral, por los accesos interlaminar parasagital y transforaminal, utilizando el Inventario Abreviado de Dolor (IAD) como herramienta de evaluación de resultados. Para la variante interlaminar parasagital se estudió también el patrón de distribución del contraste en el espacio epidural anterior y la presencia o ausencia de radiculograma, así como de parestesia o dolor homolateral durante la inyección del esteroide. También se estudió el perfil de efectos colaterales y complicaciones de la técnica.

\section{MATERIAL Y MÉTODO}

Se trata de un estudio prospectivo, aleatorizado, para evaluar el efecto de la inyección epidural de betametasona utilizando los abordajes interlaminar parasagital y transforaminal bajo radioscopia, en una población 
seleccionada con dolor radicular lumbar unilateral a causa de hernia discal, protrusiones discales lumbares o canal estrecho lumbar. Como instrumento para evaluar el dolor de los pacientes seleccionados se empleó el Inventario Abreviado de Dolor (IAD). Este cuestionario de autocumplimentación permite a los pacientes cuantificar la intensidad de su dolor y el grado en el cual este interfiere con aspectos emocionales y funcionales. Además, el cuestionario consta de ítems adicionales que evalúan el nivel subjetivo de alivio que el tratamiento proporciona, la localización del dolor y la descripción del mismo. El cuestionario se basa en preguntas vinculadas a la intensidad del dolor y en cómo este afecta a la vida de la persona en distintos aspectos, graduándose del 0 al 10. El análisis de ambas dimensiones permite obtener los Índices de Intensidad y de Interferencia. El Índice de Intensidad surge de promediar las respuestas vinculadas a la intensidad del dolor, mientras que el de Interferencia resulta de promediar las respuestas vinculadas con las repercusiones funcionales y afectivas que dicho dolor produce (10-12). La pregunta número 6 del cuestionario se refiere a la intensidad del dolor en el momento en que se aplica el cuestionario, y puede equivaler a la aplicación de la Escala Verbal Numérica (EVN), habitualmente utilizada como instrumento de evaluación de resultados. El cuestionario se aplicó previamente a la realización del procedimiento, que se consideró como situación basal, y se repitió en el control al mes de realizado el mismo.

Los criterios de inclusión en el estudio fueron: pacientes con síndrome radicular lumbosacro de 6 o más meses de evolución, con una resonancia magnética con evidencia de hernia de disco, protrusión discal o estenosis del canal, con escasa respuesta al tratamiento farmacológico durante, por lo menos, un mes. Se estableció como respuesta satisfactoria a los esteroides epidurales una disminución de 2 puntos o más en la EVN (pregunta 6 del cuestionario) al mes de realizado el último procedimiento de inyección (13).
Se tomaron como criterios de exclusión los siguientes: pacientes menores de 18 años y mayores de 80 , pacientes embarazadas y pacientes con lumbalgia sin irradiación radicular, dolor radicular lumbar bilateral y cirugía de columna previa.

El estudio fue aprobado por el Comité de Ética del Hospital de Clínicas. Todos los pacientes firmaron el formulario de consentimiento informado habitualmente utilizado para el procedimiento.

Los procedimientos fueron realizados en sala de operaciones. Con el paciente en posición decúbito ventral se colocó una vía venosa periférica. La asepsia se efectuó con clorhexidina alcohólica y la anestesia local con lidocaína al 0,5 \%. En la técnica interlaminar parasagital se utilizaron agujas de Tuohy números 18, 311/2 o 43/4 pulgadas según la complexión física del paciente. Con enfoque radioscópico anteroposterior, se introdujo la aguja en los espacios interlaminares, L4/L5 o L5/ S1, utilizando la técnica en visión túnel, parasagital homolateral del lado correspondiente al dolor radicular (Figura 1). Se realizó pérdida de resistencia con suero fisiológico. En el enfoque lateral se administró una inyección de $4 \mathrm{ml}$ de contraste (Omnipaque ${ }^{\circledR}$ ] y se consignó la presencia de epidurograma anterior, posterior o ambos (Figura 1). En el enfoque anteroposterior se consignaron las características del radiculograma y del epidurograma (Figura 1). Luego se administró un frasco de betametasona (12 mg de una mezcla de fosfato y acetato de betametasona, en 2 cc de solución] más 3 cc de lidocaína al 0,5\% (total 5 cc de solución). Se evaluó la calidad técnica del bloqueo con elementos clínicos como la presencia de parestesias o dolor concordante durante la inyección, y datos radiológicos como la presencia de radiculograma y de epidurograma anterior (Figura 1). En el grupo tratado con betametasona por vía epidural transforaminal se utilizaron agujas de Quincke números 22, 31/2 o 43/4 pulgadas con enfoques radiológicos oblicuo, lateral y anteroposterior. El punto objetivo en posición oblicua fue la zona subpedicular, en

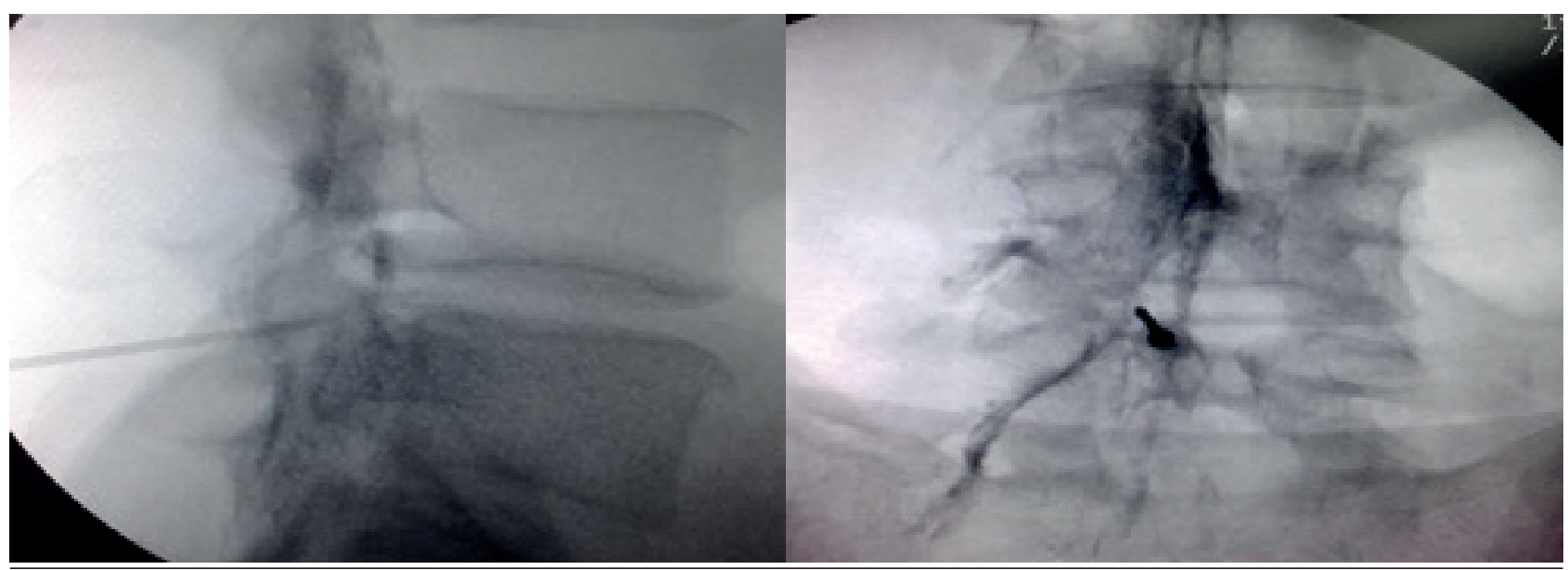

Fig. 1. Abordaje interlaminar parasagital L5-S1. A la izquierda se observa el enfoque radioscópico lateral, con la aguja de Tuohy en el espacio epidural. El contraste inyectado se distribuye por los espacios epidural anterior y posterior. A la derecha se observa el enfoque radioscópico anteroposterior, con la aguja de Tuohy lateralizada hacia la izquierda. La inyección de contraste permite observar el epidurograma en este enfoque y el radiculograma de las raíces L4, L5 y S1 izquierdas. 
enfoque lateral el ángulo anterosuperior o techo del neuroforamen, en el llamado triángulo de seguridad en el enfoque anteroposterior (Figura 2). La inyección de contraste fue equivalente a la realizada en el grupo interlaminar, debiéndose obtener radiculograma y epidurograma tras la inyección (Figura 2). La solución de betametasona fue la misma que la utilizada en el grupo interlaminar, inyectándose la totalidad de la solución por el neuroforamen elegido, o la mitad de la solución en cada neuroforamen cuando por criterios clínicos se decidió la inyección en dos niveles.

Se midieron, previamente al procedimiento y en el control a las 4 semanas, los siguientes datos: intensidad de dolor por la EVN (pregunta 6) e Índices de Intensidad e Interferencia. La determinación de una respuesta positiva o satisfactoria se estableció como un descenso de 2 puntos o más en la EVN. Se estableció el porcentaje o fracción de pacientes con respuestas positivas o negativas.

Los datos se presentan como la media y su desviación estándar. Se utilizó la prueba t de Student para muestras pareadas e independientes, según el caso, y la prueba de chi cuadrado para la evaluación estadística de proporciones. Un valor de $p<0,05$ se estableció como de significancia estadística. Las gráficas y el análisis estadístico fueron realizados con el programa Graphpad Prism versión 7.0.

\section{RESULTADOS}

Un total de 64 pacientes con lumbociatalgia unilateral fueron incluidos en el estudio en el periodo de enero del 2016 a noviembre del 2017. De ellos, 29 fueron tratados con inyecciones epidurales de esteroides por vía interlaminar parasagital, mientras que 25 fueron tratados utilizando el abordaje transforaminal. Diez pacientes que recibieron el tratamiento no volvie- ron al control (3 por vía interlaminar parasagital, 7 por vía transforaminal). La edad promedio de los pacientes fue de 51 años. En cuanto al sexo, 32 pacientes eran de sexo femenino y 8 de sexo masculino. El tiempo promedio de evolución del dolor fue de 29 meses. La totalidad de los pacientes presentaron en la resonancia magnética protrusiones discales en los espacios L4/L5 y L5/S1, con grados variables de estenosis del canal. No se trataron pacientes con hernias discales. De los 54 pacientes, 29 fueron tratados con betametasona por la vía interlaminar parasagital y 25 por la vía transforaminal. La betametasona administrada por la vía interlaminar parasagital redujo la puntuación en la EVN un $20 \%$, de 7,7 $\pm 1,5$ a 6,2 $\pm 3,1$ (diferencia estadísticamente significativa, $p=0,03$, prueba t de Student para muestras pareadas] y por la vía transforaminal un $36 \%$, de $8,3 \pm 1,5$ a $5,3 \pm$ 3,3 ( $p=0,0001)$. La Figura 3 muestra los valores de la EVN normalizados (esto es, expresados como porcentaje de cambio] de ambos grupos, basales y al mes del tratamiento. Si bien se puede observar que el porcentaje de cambio es mayor en los pacientes en los que se utilizó la vía transforaminal que en los que se utilizó el abordaje interlaminar, esta diferencia no es estadísticamente significativa $(p=0,13$, prueba t de Student para muestras independientes).

Los Índices de Intensidad e Interferencia también fueron reducidos por la inyección epidural de esteroides (Figuras 4 y 5). La betametasona administrada por la vía interlaminar parasagital redujo el Índice de Intensidad de 7,2 $\pm 1,2$ a 5,8 $\pm 2,1$ y el Índice de Interferencia de $7,0 \pm 1,8$ a $5,8 \pm 2,5$, en ambos casos una disminución estadísticamente significativa $[p=0,002$ y $p=0,01$, respectivamente; prueba t de Student para muestras pareadas). La betametasona administrada por la vía transforaminal redujo el Índice de Intensidad de 7,7 $\pm 1,6$ a 5,7 $\pm 2,4$ y el Índice de Interferencia de $7,7 \pm 1,7$ a $6,3 \pm 3$, reducción estadísticamente

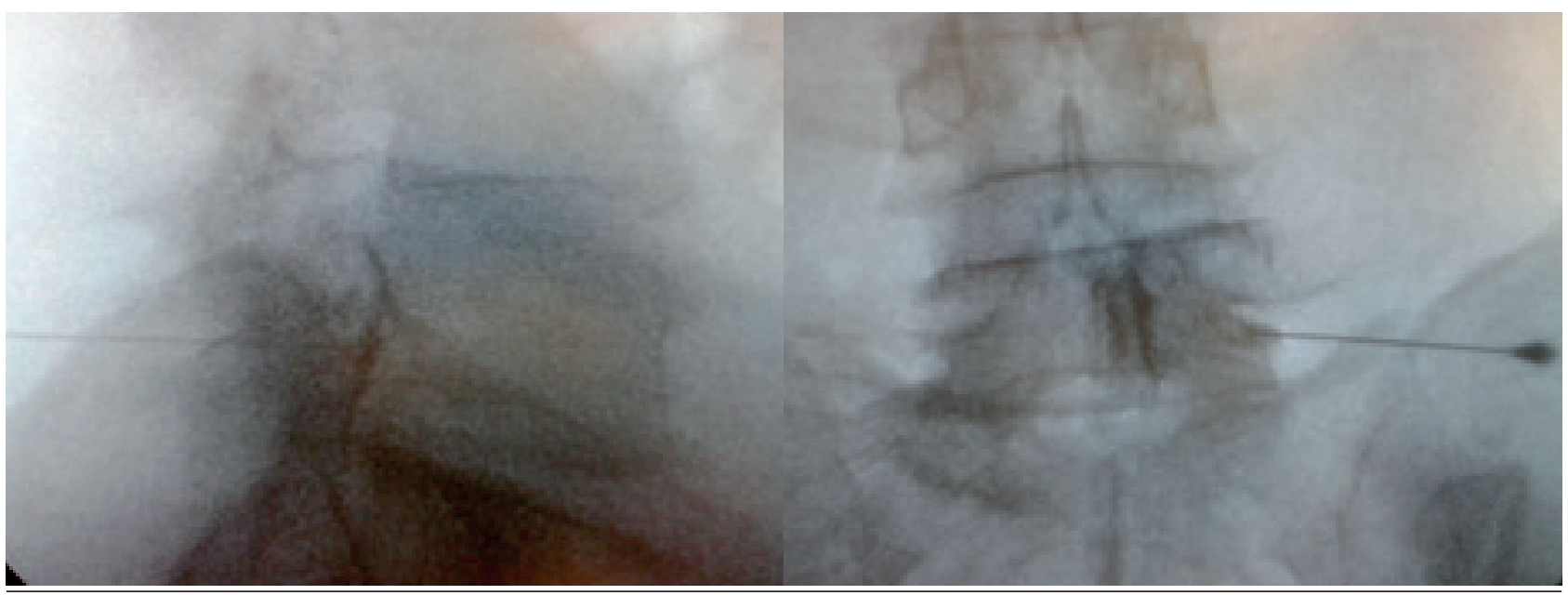

Fig. 2. Abordaje transforaminal L5-S1 derecho. A la izquierda se observa la aguja de Quincke con su extremo localizado en el ángulo anterosuperior del neuroforamen correspondiente. La inyección de contraste dibuja el espacio epidural anterior. A la derecha se observa el enfoque radioscópico anteroposterior, con el correspondiente epidurograma y radiculograma de la raíz L5 derecha. 


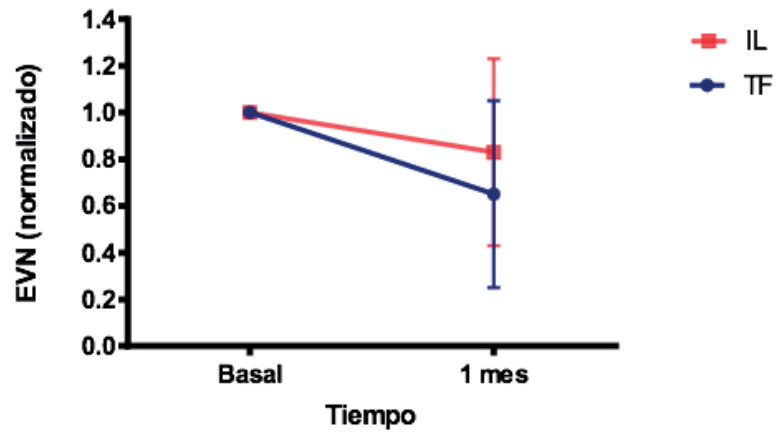

Fig. 3. Variación de las puntuaciones en la Escala Verbal Numérica (EVN) con respecto a los valores basales como datos normalizados, al mes de las inyecciones epidurales de betametasona por los accesos interlaminar (IL, $n=29$ ) $y$ transforaminal (TF, $n=25$ ). No existen diferencias significativas en las variaciones de la EVN entre ambos accesos [prueba t de Student para muestras independientes].

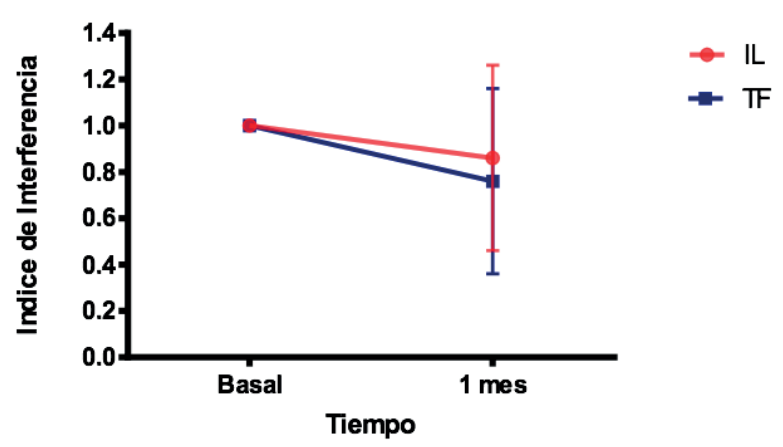

Fig. 5. Variación de las puntuaciones en el Índice de Interferencia con respecto a los valores basales como datos normalizados, al mes de las inyecciones epidurales de betametasona por los accesos interlaminar (IL, $n=29$ ) y transforaminal (TF, $n=25$ ). No existen diferencias significativas en las variaciones en el Índice de Interferencia entre ambos accesos (prueba t de Student para muestras independientes].

significativa $(p=0,0002$ y $p=0,008$, respectivamente). No hubo diferencias estadísticamente significativas entre ambos accesos al comparar la reducción de ambos índices.

El porcentaje de respuestas satisfactorias o positivas fue mayor en el grupo transforaminal cuando se lo comparó con el acceso interlaminar parasagital. La proporción de respuestas satisfactorias en los accesos interlaminar parasagital y transforaminal fue de 11/29 (38\%) y 16/25 (64\%) respectivamente, diferencia estadísticamente significativa con un valor de $p=0,01$ (prueba de Chi cuadrado).

Cuando se compararon los cambios en la EVN y en el Î́ndice de Interferencia de aquellos pacientes cuyas

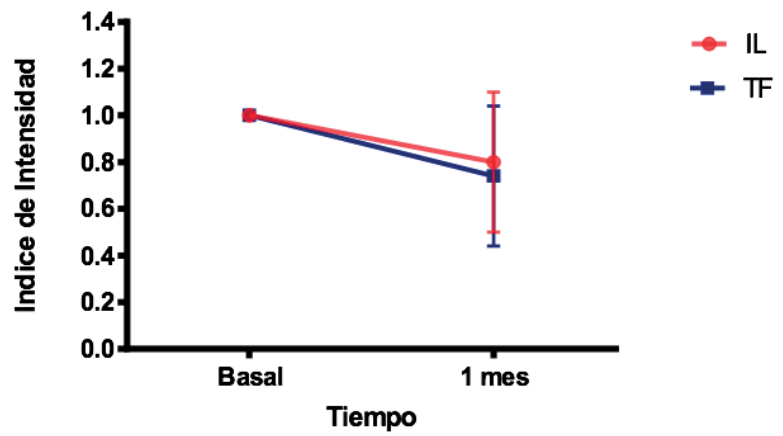

Fig. 4. Variación de las puntuaciones en el Índice de Intensidad con respecto a los valores basales como datos normalizados, al mes de las inyecciones epidurales de betametasona por los accesos interlaminar ( $\mathrm{L}, \mathrm{n}=29$ ) y transforaminal (TF, $n=25$ ]. No existen diferencias significativas en las variaciones del Índice de Intensidad entre ambos accesos [prueba t de Student para muestras independientes].

respuestas se consideraron satisfactorias utilizando betametasona por vía interlaminar o parasagital (11 y 16 respectivamente), no se observaron diferencias en ninguno de estos parámetros (Figuras 6 y 7).

La puntuación en la EVN de los pacientes tratados con betametasona por vía interlaminar cambió de $8,4 \pm 1,4$ a $3,2 \pm 3$, lo que equivale a un $61 \%$ de mejoría, mientras que el Índice de Interferencia cambió de $6,5 \pm 2,2$ a $4,5 \pm 2$, lo que equivale a un $30 \%$ de mejoría. En el caso de la betametasona administrada por vía transforaminal, la EVN pasó de 8,7 $\pm 1,1$ a $3,3 \pm 2,3$, lo que equivale a un $63 \%$ de mejoría, mientras que el Índice de Interferencia pasó de 7,13 $\pm 2,1$ a $4,75 \pm 3,1$, lo que equivale a un $33 \%$ de mejoría.

La Figura 6 muestra los cambios observados en la intensidad del dolor en los pacientes que presentaron respuestas satisfactorias en ambos grupos, expresados como porcentaje de cambio. En ambos grupos los pacientes pasaron de padecer un dolor intenso a uno leve. Por lo tanto, tomando por separado al grupo de "respondedores", el dolor pasó de intensidad elevada a leve en ambos grupos, con una mejoría de 2 puntos en cuanto a la repercusión funcional del dolor medido con el Îndice de Interferencia (Figura 7). No se observaron diferencias estadísticamente significativas en los resultados obtenidos comparando ambos accesos cuando se toman por separado los pacientes con respuestas positivas.

En 21 de los 26 procedimientos realizados por el acceso interlaminar parasagital se evaluó la calidad técnica de la inyección epidural de esteroides. En 15 (71\%) se obtuvo epidurograma anterior, esto es, alcance de la solución en la cara ventral del espacio (Figura 1 izda.); en 17 (85\%) el paciente refirió dolor o parestesias en el territorio radicular durante la inyección, y en 12 (60 \%) se observó radiculograma junto a la imagen característica de epidurograma.

No se consignaron complicaciones en ninguno de los pacientes tratados. 


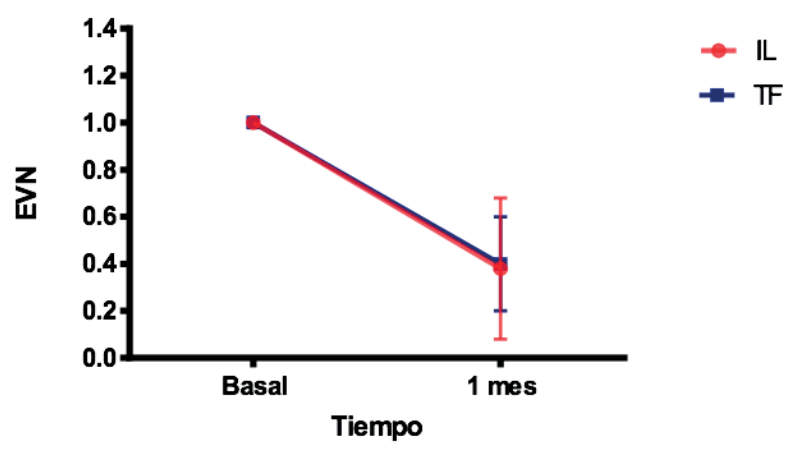

Fig. 6. Variación de las puntuaciones en la Escala Verbal Numérica [EVN] con respecto a los valores basales como datos normalizados, al mes de las inyecciones epidurales de betametasona por los accesos interlaminar (IL, $n=11$ ) y transforaminal (TF, $n=16$ ), incluyendo únicamente los pacientes considerados como respondedores (variación en 2 puntos de la EVN con respecto al valor basal). No existen diferencias significativas en las variaciones de la EVN entre ambos accesos (prueba t de Student para muestras independientes].

\section{DISCUSIÓN}

Este estudio es, hasta donde sabemos, el primero en comparar las vías de acceso epidural interlaminar y transforaminal utilizando el IAD como herramienta de valoración de resultados. Este inventario se utiliza en nuestro Servicio desde hace 5 años: se trata de una herramienta útil para evaluar los resultados de los tratamientos analgésicos que se realizan (11).

Tomando en conjunto los pacientes respondedores y no respondedores a una inyección única del esteroide betametasona en el espacio epidural lumbar por las vías interlaminar parasagital y transforaminal, resultó en una discreta mejoría, pero estadísticamente significativa, de la intensidad del dolor y la repercusión funcional de este en pacientes con síndrome radicular lumbosacro crónico unilateral, evaluados por el IAD. No existieron diferencias estadísticamente significativas en estos datos respecto a la administración de betametasona si se comparan ambos accesos.

Es bien conocido que existen pacientes que, por distintos factores, no tienen repuestas satisfactorias a las inyecciones de esteroides administrados por cualquier vía de acceso. Factores como largos tiempos de evolución y aspectos psicológicos previos como ansiedad, depresión o elevados niveles de catastrofismo en la percepción de la sensación dolorosa influyen en las respuestas analgésicas satisfactorias (14-16).

Asimismo, el tipo de lesión encontrada en los estudios de imagen puede influir en una respuesta menor, dado que las mejores respuestas analgésicas de las inyecciones de esteroides se ven en pacientes con hernias discales [5].

En nuestra muestra los pacientes presentaron un tiempo de evolución del dolor mayor de 2 años, siendo las lesiones encontradas en la mayoría de los casos protrusiones discales lumbares y grados variables de estenosis del canal, y en ningún caso hernias de disco. No fue evaluado el papel de factores psicológicos como

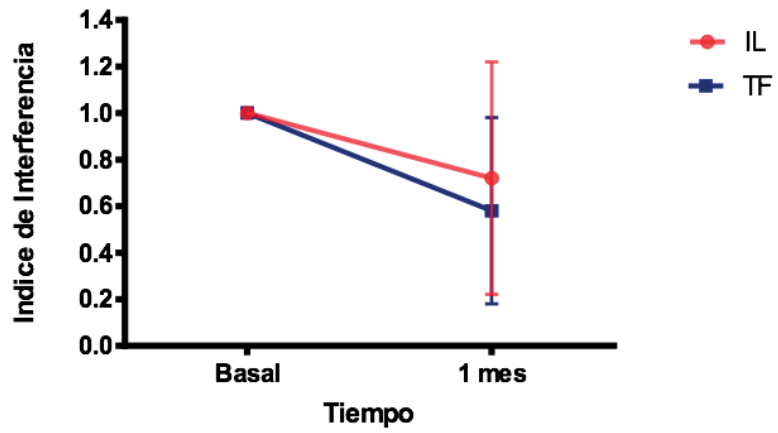

Fig. 7. Variación de las puntuaciones en el Índice de Interferencia con respecto a los valores basales como datos normalizados, al mes de las inyecciones epidurales de betametasona por los accesos interlaminar (IL, $n=11$ ) y transforaminal (TF, $n=16$ ), incluyendo únicamente los pacientes considerados como respondedores (variación en 2 puntos de la EVN con respecto al valor basal). No existen diferencias significativas en las variaciones en el Índice de Interferencia entre ambos accesos [prueba t de Student para muestras independientes].

depresión, ansiedad o catastrofismo en el porcentaje de respuestas exitosas a las inyecciones de esteroides, siendo tal evaluación parte de un estudio curso.

Cuando se discriminan aquellos pacientes cuya intensidad del dolor se reduce 2 puntos en la EVN, y se separan respondedores de no respondedores, encontramos que la inyección epidural por vía interlaminar parasagital muestra un $38 \%$ de respuestas clínicamente aceptables frente a un $64 \%$ cuando la betametasona se inyecta por vía transforaminal, diferencia estadísticamente significativa. Esto refleja una tendencia a una respuesta analgésica más eficaz cuando se utiliza la betametasona administrada por vía transforaminal que cuando se utiliza por vía interlaminar.

Si bien estos porcentajes de éxito son algo inferiores a los encontrados en otros estudios sobre el tema, que oscilan entre el $75 \%$ y el $80 \%$ de eficacia, nuestros resultados se refieren al éxito de una inyección única, mientras que en las referencias señaladas es frecuente que se evalúen los resultados de inyecciones repetidas de esteroides (5).

Aquellos pacientes que fueron clasificados como "respondedores" o con respuestas satisfactorias utilizando la EVN presentaron un cambio clínicamente significativo en la intensidad del dolor y su repercusión funcional. En ellos, el dolor pasó de un nivel de intensidad catalogado como intenso (8-9 en la EVN) a un dolor de leve intensidad ( 3 en la EVN) con un descenso de por lo menos 2 puntos en el Índice de Interferencia. En este grupo de pacientes la mejoría observada no mostró diferencias clinicas ni estadísticamente significativas según el acceso utilizado para la inyección epidural.

La administración epidural de esteroides utilizando los accesos estudiados en este trabajo ha sido evaluada por otros autores. Candido, en el año 2008, describe la técnica de abordaje interlaminar parasagital y encuentra una eficacia similar a la observada utilizando la vía transforaminal, hallazgo que es luego reproducido por otros autores, por lo que se propone como una técnica 
alternativa a la vía transforaminal pero sin sus riesgos neurológicos $(17,18)$.

Un factor posible para explicar el porcentaje menor de respuestas aceptables utilizando el acceso interlaminar parasagital en nuestro estudio es el uso de un volumen menor de solución (5 cc) al corrientemente utilizado en este acceso (8 a $10 \mathrm{cc}$ ). Sin embargo, un volumen de 2,5 a 5 cc es el habitualmente usado en la vía transforaminal, lo que podría explicar una mayor disponibilidad del esteroide en la zona de conflicto discorradicular. El empleo de mayores volúmenes de solución inyectados en el espacio epidural se relaciona con mejores respuestas analgésicas que cuando se utilizan volúmenes menores, probablemente por un efecto de dilución y/o lavado de sustancias algogénicas del espacio epidural (19).

Otro factor posible para explicar un menor porcentaje de éxito con la vía interlaminar parasagital es el alcance al sector anterior del espacio epidural observado (75\%). En el estudio realizado por Candido, este acceso mostró un $100 \%$ de alcance al espacio epidural anterior, incluso mayor al observado cuando se utiliza la vía transforaminal (75 \%), lo que no pudo ser reproducido en el presente estudio (17).

\section{CONCLUSIÓN}

La inyección epidural lumbar única de betametasona en pacientes con síndrome radicular lumbar produjo una reducción clínicamente significativa a corto plazo de la intensidad del dolor y su repercusión funcional en aproximadamente la mitad de los pacientes tratados. La inyección del esteroide por vía transforaminal mostró con más frecuencia ser más efectiva que administrado por vía interlaminar parasagital. Las técnicas resultaron seguras, no consignándose complicaciones o efectos colaterales importantes.

\section{CONFLICTO DE INTERESES}

Los autores declaran no tener ningún conflicto de intereses.

\section{BIBLIOGRAFÍA}

1. Castromán P, Surbano M, Alberti M, Schwartzmann A, Cristiani F, Ayala S. Inyección epidural de corticoides en el tratamiento del Sindrome Radicular Lumbosacro (SRL). Anest Analg Reanim [Internet]. 2015;28(2):3-3. Disponible en: http:// www. scielo.edu. uy/scielo. php?script=sci_arttext\&pid=S168812732015000200004\&ling=es.

2. Knezevic NN, Lissounov A, Candido KD. Adding particulate or non-particulate steroids to the local anesthetics when performing parasagittal interlaminar epidural injections. Pain Physician. 2014;17:E633-E671.

3. Manchikanti L, Singh V, Cash KA, Pampati V, Falco FJ. The role of fluoroscopic interlaminar epidural injections in managing chronic pain of lumbar disc herniation or radiculitis: A randomized, double-blind trial. Pain Practice. 2013;13:547-58.

4. Manchikanti L, Singh V, Cash KA, Falco FJ, Pampati V. Evaluation of the effectiveness of lumbar interlaminar epidural injections in managing chronic pain of lumbar disc herniation or radiculitis: a randomized, double-blind, controlled trial. Pain Physician. 2010;13:343-55.

5. Manchikanti L, Abdi S, Alturi S, Benyamin RM, Boswell MV, Buenaventura RM, et al. An update of comprehensive evidence-based guidelines for interventional techniques in chronic spinal pain. Part II: guidance and recommendations. Pain Physician. 2013;16(2 Suppl):S49-283.

6. Manchikanti L, Pampati V, Falco FJE, Hirsch JA. Assesment of the growth of epidural injections in the Medicare population from 2000 to 2011. Pain Physician. 2013;16:E349-E364.

7. Manchikanti L, Cash KA, Pampati V, Falco FJE. Transforaminal epidural injections in chronic lumbar disc herniation: a randomized, double-blind, active controlled trial. Pain Physician. 2014;17:E489-E501.

8. Rathmell JP, Benzon HT, Dreyfuss P, Huntoon M, Wallace M, Baker R, et al. Safeguards to prevent neurologic complications after epidural steroid injections. Consensus opinions from a Multidisciplinary Working Group and National Organizations. Anesthesiology. 2015; 122(5):974-84.

9. Benzon HT, Chew TL, McCarthy RJ, Benzon HA, Walega DR. Comparison of the particle sizes of different steroids and the effect of dilution: a review of the relative neurotoxicities of the steroids. Anesthesiology. 2007;106(2):331-8.

10. Keller S, Bann C, Dodd SH, Schein J, Mendoza T, Cleeland $\mathrm{CH}$. Validity of the Brief pain Inventory for use in documenting the outcomes of patients with non-cancer pain. Clin J Pain. 2004;20(5):309-18.

11. Surbano M, Antúnez M, Coutinho I, Machado V, Castroman P. Uso del Brief Pain Inventory (BPI) para la evaluación de las técnicas intervencionistas en el tratamiento de la lumbalgia. Revista El Dolor. 2014(62):10-4.

12. Roland M, Fairbank J. The Roland-Morris Disability Questionnaire and the Owestry Disability Questionnaire. Spine. 2000;25(24):3115-24

13. Gatchel RJ, Mayer TG Choi YH, Chou CR. Validation of a consensus-based minimal clinically important difference (MCID) threshold using an objective functional external anchor. Spinal J. 2013;13(8):889-93.

14. Sivaganesan A, Chotai S, Parker SL, Asher AL, McGirt MJ, Devin CJ. Predictors of the efficacy of epidural steroid injections for structural lumbar degenerative pathology. Spine J. 2016;16(8):928-34.

15. Samwel H, Sappendel R, Crul BJP, Voerman VF. Psychological predictors of the effectiveness of radiofrequency lesioning of the cervical spinal dorsal root ganglion. Eur $\mathrm{J}$ Pain. 2000;4:149-55.

16. Turner JA, Comstock BA, Standaert CHJ, Heagerty PJ, Jarvic JG, Deyo RA, et al. Can patient characteristics predict benefit from epidural corticosteroid injections for lumbar spinal stenosis symptoms? Spine J. 2015;15:2319-31.

17. Candido KD, Raghavendra MS Chintagada M, Badiee S, Trepashko DW. A prospective evaluation of iodinated contrast flow patterns with fluoroscopically guided lumbar epidural steroid injections: the lateral parasagittal interlaminar epidural approach versus the transforaminal epidural approach. Anesth Analg. 2008;106:638-44.

18. Chang Chien GC, Knezevic NN, McCormick Z, Chu SK, Trescot AM, Candido KD. Transforaminal versus interlaminar approaches to epidural steroid injections: a systematic review of comparative studies for lumbosacral radicular pain. Pain Physcian. 2014;17:E509-E524.

19. Rabinovitch DL, Peliowski A, Furlan AD. Influence of lumbar epidural injection volume on pain relief for radicular leg pain and/or low back pain. Spine J. 2009;9:509-17. 